\title{
Yokohama: MATSim Application for Resilient Urban Design
}

\author{
Yoshiki Yamagata, Hajime Seya and Daisuke Murakami
}

\subsection{Introduction}

In Yamagata and Seya (2015), we proposed the concept of a resilient local electricity-sharing system as a complement, or alternative, to a FIT (feed-in tariff) to achieve $\mathrm{CO}_{2}$-neutral transportation in cities. In our proposed system, electricity generated from widely introduced solar PVs (Photovoltaic Panels) is stored in cars "not in use" in a city. In Japan, almost half the central Tokyo metropolitan area cars are used only on weekends and thus are kept parked weekdays. These cars could represent a huge new storage potential if they were replaced by EVs; that is, they could be used as storage batteries in a V2C (Vehicle to Community) system.

This study analyzed the potential of EVs as storage batteries in emergency cases. Specifically, we focused on the following three questions:

1. How much residential demand can be met (in each 24 hour) by electricity from just PVs, which are installed on the roofs of all detached houses in the study area?

2. How many EVs are needed to store all surplus electricity (PV supply minus demand)?

3. How does EVs driving change the load curve and how can mass-adopted PVs fulfill total demand?

To answer our second and third questions, we needed to know (a) the number of cars parked at home during each hour (that is, the time each car arrived at home after use) and (b) the amount of battery charge consumed by each driver during his/her daily trips (that is, trip duration). For this simulation, we used MATSim. In this chapter, we briefly introduce our MATSim application for a local electricity-sharing system in Yokohama city, based on Yamagata and Seya (2013); Yamagata et al. $(2014,2015)$. 


\subsection{Results}

We assumed that PV was installed on the roof of each detached house in Yokohama city. Then, we calculated the amount of electricity supplied each hour throughout the whole day by employing simple intensity method. The O-D trip data used are from the Fourth Person Trip Survey in Tokyo Metropolitan Area, implemented in 1998. The data are available through the People Flow Project (http://pflow.csis.u-tokyo.ac.jp) on request (application) and include the O-D trips by traffic mode, time of day, purpose, etc. for each micro district, called cho-cho-moku. The Person Trip survey is a national survey that focuses on people's travel behavior during a given few days of each month, from October to December. Because the number of cars in Yokohama for each chocho-moku was unknown, the city-level value was allocated to the cho-cho-moku (areal weighting) and adjusted for the size of the population. The road-network information was taken from the National Digital Road Map Database and included sufficient data on road capacity, width classification, link length, number of lanes and travel speed to perform traffic simulations in MATSim. MATSim requires a daily "plan file" for each agent (car driver); we prepared these files by using the Fourth Person Trip Survey, which captured the daily movements of 722000 people. Because the Fourth Person Trip Survey sampled approximately $2 \%$ of the population of the Tokyo metropolitan area, the plan file was replicated according to the intensity factor provided by the People Flow Project, resulting in 505335 agents. From the MATSim simulation, we had obtained each agent's trip duration and arrival time.

Considering load curve changes due to the EVs driving, we then asked if massively adopted PVs would be enough to satisfy total energy demand in Yokohama. In Figure 96.1, the solid and dashed lines represent electricity surplus cumulative distribution, charged to or discharged from the batteries of EVs, not in use and used only for charging the EVs in use, during May and August (solid line, maximum; dashed line, average). The dotted line in the figure represents the scenario where electricity surplus was both charging EVs and satisfying households' typical electricity demand

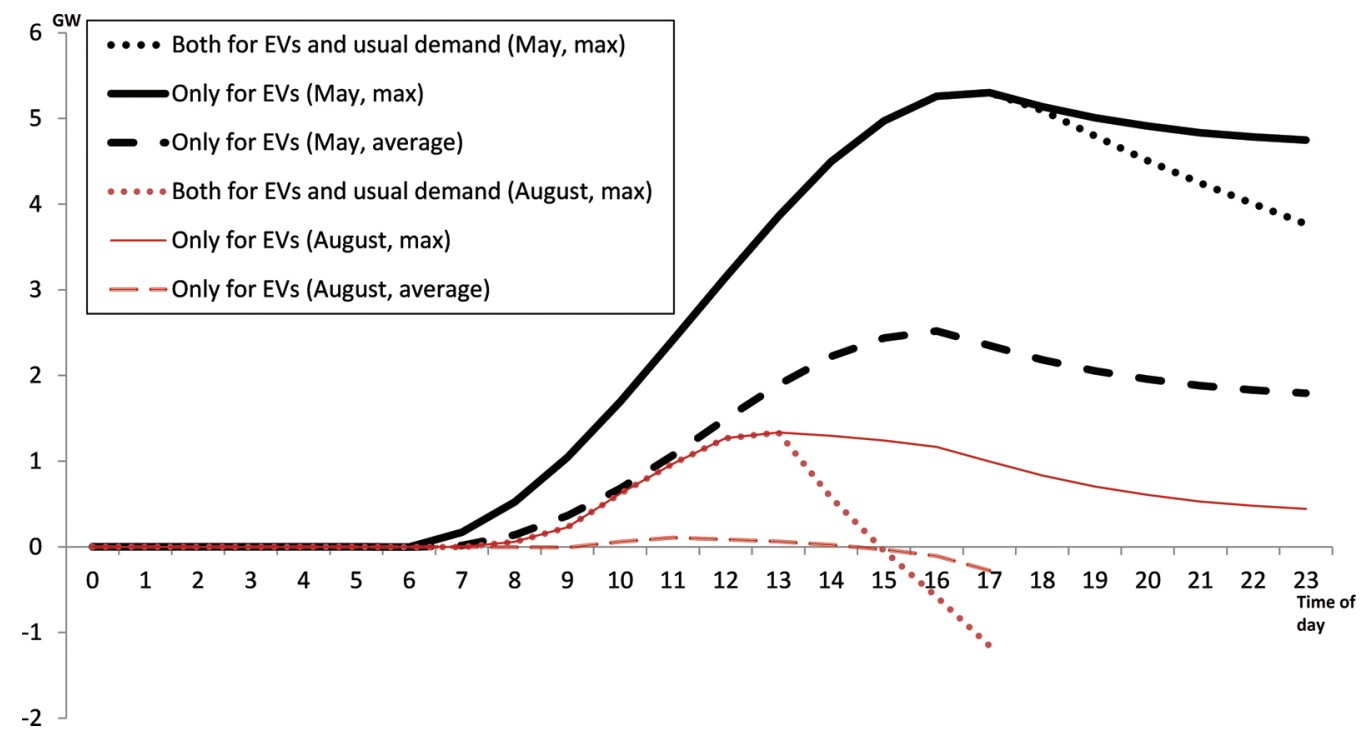

Figure 96.1: Cumulative distribution of electricity surplus charged to or discharged for electricity demand ( $y$ axis denotes the cumulative distribution of electricity surplus).

Source: Reproduced by permission of the Institution of Engineering \& Technology: published in Yamagata and Seya (2015, Figure 6) 
under maximal/average solar irradiance. However, in August (high demand, high PV supply), the electricity surplus was sufficient for charging EVs, but not enough to meet the households' huge electricity demand due to evening use of air-conditioning.

To meet household electricity demand, PV electricity needs be efficiently stored in EVs and locally shared. For example, if a high-affordability zone (storage capacity is greater than electricity surplus) is adjacent to a low-affordability zone (storage capacity is smaller than electricity surplus), then the share of their EV capacity increases the ratio of stored PV electricity. Because storage affordability (storage capacity minus electricity surplus) is significantly different regionally (see Figure 96.2), clustering of community-based local sharing must be carefully designed. In this study, we attempted to optimize community clusters using several different algorithms. Firstly, the number of clusters was assumed 18 to be the same as the number of Yokohama city wards. Then, cluster optimization was performed by minimizing (the sum of storage affordability in the 18 clusters) plus $k$ (minimum circularity in these clusters), where $k$ was the weight for the circularity. The first term balanced storage capacity and electricity surplus to increases the rate of stored PV electricity; the second term decreased inter-point distance within each cluster, as well as electricity sharing (transmission) cost. The minimization was conducted in every month through a simulated annealing algorithm to find optimal spatially clustered communities.

Figure 96.3 shows four-month clustering results; all clusters indicate positive storage affordability in April, May, June, July, September, and October. In other words, PV electricity covers whole household electricity demands, if EV capacities are shared with these optimized clusters.

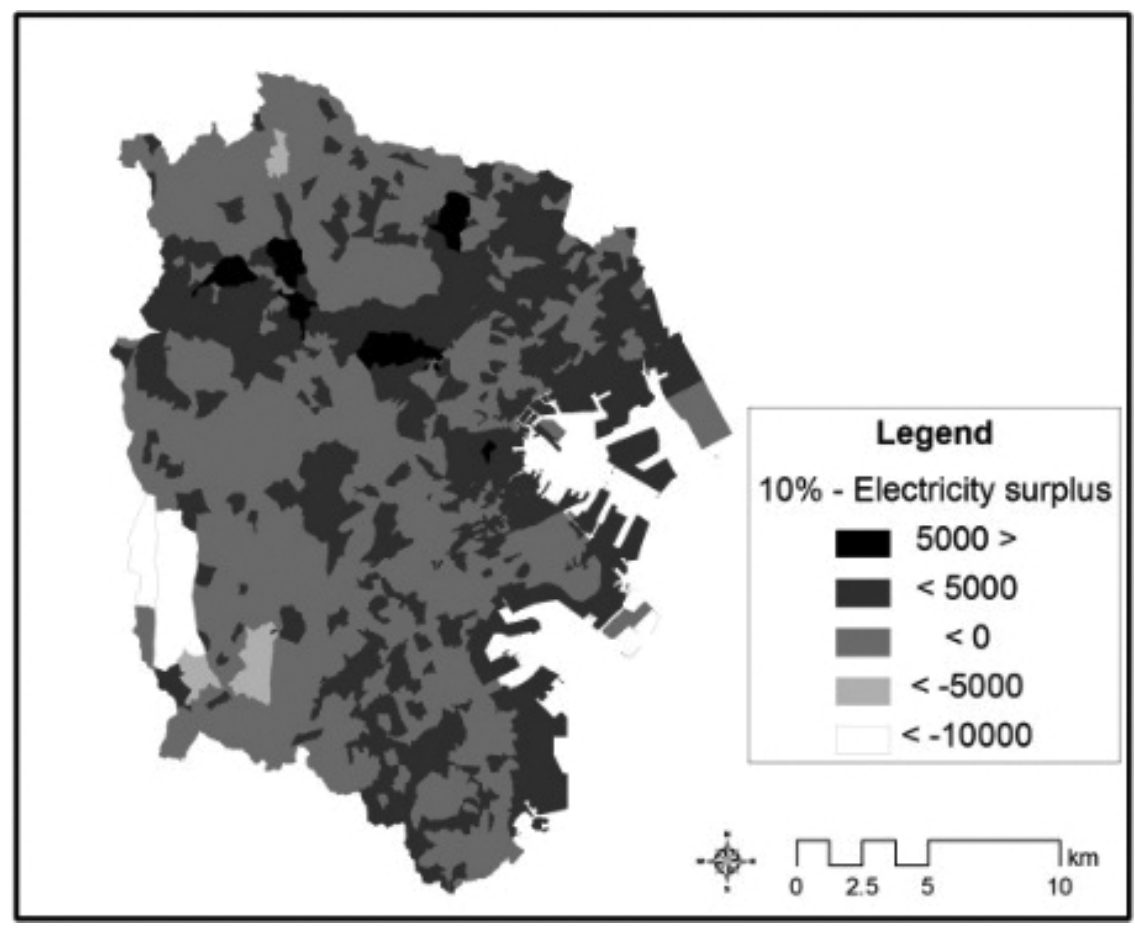

Figure 96.2: Storage affordability: Storage capacity minus electricity surplus in kWh/day (10 \% of EVs not in use being used as battery).

Source: Reproduced by permission of the Institution of Engineering \& Technology: published in Yamagata and Seya (2015, Figure 10.a) 


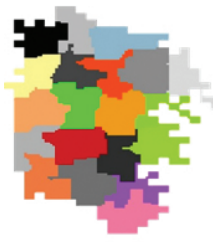

March

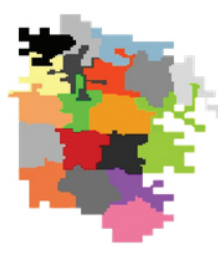

June

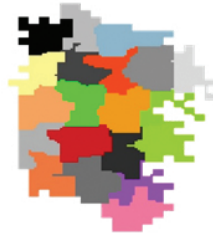

September

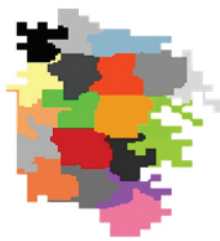

December

Figure 96.3: Monthly clustering results.

In summary, we applied MATSim to analyze the potential of EVs in a V2C system and found that EVs can cover typical household electricity demands in some months and the cover ratio can be increased by community clustering for local electricity sharing. In the future study, we plan to use MATSim to simulate mobility behavior for electricity sharing community scenarios and extend our clustering analysis utilizing simulated behavior. Finally, development of community level mobility sharing service would be a very important topic to integrate MATSim simulations with our land use and transportation scenarios, such as compact and dispersion scenarios (see Yamagata et al., 2013). 